\title{
FROM CIRCUITS TO SPECTRAL PEAKS
}

\author{
J.H.B. Deane \\ School of Electronic Engineering, Information Technology and Mathematics, \\ University of Surrey, \\ Guildford GU2 5XH, \\ United Kingdom
}

\begin{abstract}
A class of clocked/autonomous circuits is defined in which the behaviour is described by a one-dimensional mapping. For these circuits, the power density spectrum at the harmonics of the clock frequency can be calculated directly from the invariant density of the mapping. An algorithm for carrying out this calculation is derived and illustrated, using the clocked monostable multivibrator as an example. An application to EMC improvement is suggested and possible extensions to the work are discussed.
\end{abstract}

\section{INTRODUCTION}

The possibility of using chaos in a switch-mode power supply to improve its electromagnetic compatibility (EMC) was raised in [1] and taken further in [2]. Our thesis there was that, rather than concentrating energy in peaks, corresponding to periodic operation, chaotic behaviour leads to a broadband spectrum which would in turn have favourable implications for EMC.

In [2] we showed how to calculate the periodic spectral components, i.e. the power density spectrum (PDS) at the clock frequency and its harmonics, in a chaotically operating boost converter. Certain simplifying assumptions were made, for example that the exact 2-D mapping [3] can be approximated as a 1-D mapping. We verified our calculations expermentally.

In this paper we describe how to calculate the periodic spectral components in a class of systems that are clocked, autonomous and one-dimensional (1-D). After defining the class of systems for which this is possible, we briefly summarise the derivation of the method used. We then present results for chaotic behaviour in a clocked monostable multivibrator to illustrate the calculation and discuss to what degree the calculation can be carried out in closed form.

\section{THE SPECTRAL CALCULATION}

The spectral calculation we now describe applies to systems which are autonomous, $1-\mathrm{D}$ and clocked $(\operatorname{period} T)$ ). For

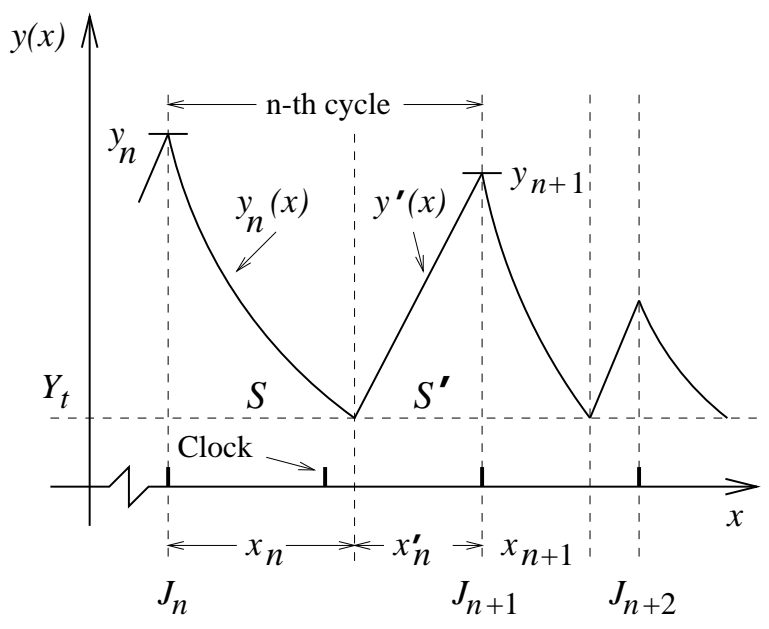

Figure 1: The waveform of a two-state clocked/thresholding system.

convenience we use rescaled time $x=t / T$ and frequency $\Omega=2 \pi f T$ (where $f$ is the linear frequency). The $n$-th cycle consists of the following steps (see figure 1):

- Initially: in state $S$, in which case the state variable as a function of rescaled time $x$ is $y_{n}(x) ;(x=0$ at the beginning of the cycle). Clock pulses are ignored.

- At time $x_{n}$ later: $y_{n}\left(x_{n}\right)=Y_{t}$, a threshold value, when the system switches to state $S^{\prime}$. The state variable is now given by $y_{n}^{\prime}(x)$. At the start of $S^{\prime}, x$ is reset to 0 .

- At the arrival of the next clock pulse, a time $x_{n}^{\prime}$ later: system switches back to state $S$.

Note that $x_{n}^{\prime}=1-x_{n} \bmod 1$ and $x_{n}+x_{n}^{\prime}=\left\lceil x_{n}\right\rceil$. To compute the periodic spectral components we need to find the Fourier transform of $R_{y}(\tau)$, the autocorrelation function of $y(x)$ : 


$$
R_{y}(\tau)=\lim _{T_{0} \rightarrow \infty} \frac{1}{T_{0}} \int_{0}^{T_{0}} y(x) y(x+\tau) d x
$$

The line of argument advanced in [2] then runs as follows:

- $y(x)=p(x)+c(x)$, where $p$ and $c$ are the periodic and non-periodic components respectively.

- The periodic component can be expressed as a Fourier series:

$$
p(x)=\sum_{m=-\infty}^{\infty} A_{m} \mathrm{e}^{j 2 \pi m x}
$$

with $A_{m}=A(2 \pi m)$, where, for any $\Omega$,

$$
A(\Omega)=\lim _{T_{0} \rightarrow \infty} \frac{1}{2 T_{0}} \int_{-T_{0}}^{T_{0}} y(x) \mathrm{e}^{-j \Omega x} d x
$$

- The autocorrelation function of $y, R_{y}(\tau)=R_{p}(\tau)+$ $R_{c}(\tau)$, i.e. the cross-correlation $R_{p c}(\tau)=0$.

- The PDS is the Fourier transform of $R_{y}(\tau)$. The periodic spectral components, from the Fourier transform of $R_{p}(\tau)$, are $\left|A_{m}\right|^{2}$ [4, Theorems 11.9 and 11.10].

Hence, we need to calculate

$$
\begin{array}{r}
A(\Omega)=\frac{1}{\left\langle X_{0}\right\rangle} \lim _{N \rightarrow \infty} \frac{1}{N} \sum_{n=1}^{N} \int_{0}^{x_{n}} y_{n}(x) \mathrm{e}^{-j \Omega\left(J_{n}+x\right)} d x+ \\
\int_{0}^{x_{n}^{\prime}} y_{n}^{\prime}(x) \mathrm{e}^{-j \Omega\left(J_{n}+x+x_{n}\right)} d x
\end{array}
$$

where the integers $J_{n+1}=\sum_{i=1}^{n}\left\lceil x_{i}\right\rceil$ with $J_{1}=0$, and $\left\langle X_{0}\right\rangle=\lim _{n \rightarrow \infty} J_{n} / n$ is the mean cycle length.

For the clock frequency and its harmonics, $\Omega=2 \pi m$, $m$ integer, and equation (1) simplifies to

$$
\begin{gathered}
A_{m}=\frac{1}{\left\langle X_{0}\right\rangle} \lim _{N \rightarrow \infty} \frac{1}{N} \sum_{n=1}^{N} \int_{0}^{x_{n}} y_{n}(x) \mathrm{e}^{-j 2 \pi m x} d x+ \\
\int_{0}^{1-x_{n} \bmod 1} y_{n}^{\prime}(x) \mathrm{e}^{-j 2 \pi m\left(x+x_{n}\right)} d x
\end{gathered}
$$

The fact that the system is autonomous and 1-D means that $y_{n}(x)$ and $y_{n}^{\prime}(x)$ are monotonic functions of a single initial condition. The implications of this are that:

1. The function $y_{n}(x)$ is invertible, and we can therefore write it as $y\left(y_{n}, x\right)$ or $y\left(x_{n}, x\right)$. It is best to choose $y_{n}(x)=y\left(x_{n}, x\right)$. The 1-D nature of the system and the fact that $y_{n}^{\prime}(0)=Y_{t}$ implies that $y_{n}^{\prime}(x)$ does not depend on $n$, and so we write $y_{n}^{\prime}(x)=y^{\prime}(x)$.
2. There is a function $F(x)$ such that $x_{n+1}=F\left(x_{n}\right)$. This function can be written down explicitly if $y$ is invertible explicitly. This is the case, for instance, when the system in state $S$ is linear.

The minimum value of $F(x)$ will be 0 ; let its maximum be $X$. If $F$ is an expanding mapping and has normalised invariant density $\rho(x)$ on $[0, X)$ then Birkhoff's Ergodic Theorem [5] states that

$$
\lim _{N \rightarrow \infty} \frac{1}{N} \sum_{n=1}^{N} \phi\left(F^{[n-1]}(x)\right)=\int_{0}^{X} \rho(u) \phi(u) d u
$$

for almost all initial conditions $x$ and for any integrable function $\phi$ which maps $[0, X)$ to $\mathcal{R}$. Here, $F^{[i]}(x)$ is the $i$-th iterate of $F$. The theorem can be used to evaluate the sum in equation (2) as

$$
\begin{array}{r}
A_{m}=\frac{1}{\left\langle X_{0}\right\rangle} \int_{0}^{X} \rho(u)\left[\int_{0}^{u} y(u, x) \mathrm{e}^{-j 2 \pi m x} d x+\right. \\
\left.\int_{0}^{1-u \bmod 1} y^{\prime}(x) \mathrm{e}^{-j 2 \pi m(u+x)} d x\right] d u
\end{array}
$$

The ergodic theorem can also be used to find $\left\langle X_{0}\right\rangle$, the mean cycle length, which is

$$
\left\langle X_{0}\right\rangle=\int_{0}^{X} \rho(x)\lceil x\rceil d x
$$

Equations (4) and (5) together can be used to calculate $\left|A_{m}\right|^{2}$, which is the PDS at the $m$-th harmonic of the clock frequency.

\section{EXAMPLES}

Several circuits can be modelled as described above. For instance, current-mode controlled buck, boost and buck-boost converters when the smoothing capacitor is large, leading to approximately $1-\mathrm{D}$ behaviour, come into this category. All the details, including spectral calculations, are in [2] for the boost converter, but what is said there applies to the other two topologies merely by redefining the parameter $\alpha$ that appears in the mapping $F$. The definitions of $\alpha$ are given in the table below; $V_{i}$ and $V_{o}$ are the input and mean output voltages respectively. The functions $y_{n}(x) / y^{\prime}(x)$, representing the inductor current, are rising/falling linear ramps.

A circuit that also comes into this category is the astable multivibrator. This has been treated as an example of chaotic behaviour in [6], although its spectral properties have not been discussed.

The repetitively-triggered monostable multivibrator see figure 2 - can also be modelled in the same way and we 
now calculate $\left|A_{m}\right|^{2}$ for this circuit. An analysis of chaotic monostable behaviour, spectral properties apart, is presented in [7]. In this case, $y\left(x_{n}, x\right)=\mathrm{e}^{\beta\left(x_{n}-x\right)}-1$ and $y^{\prime}(x)=$ $1-\mathrm{e}^{-\gamma x}$, with $y$ and $y^{\prime}$ representing the rescaled timing capacitor voltage, $\left(v_{A}-v_{B}\right) / V_{c c}$. The parameters are $\beta=$ $T /\left(R_{1} C\right)$ and $\gamma=T /\left(R_{2} C\right)$ where $T$ is the clock period.

The mappings $F$ for the circuits are given below.

\begin{tabular}{|c|c|}
\hline Circuit & Mapping, $F(x)$ \\
\hline $\begin{array}{l}\text { Buck converter } \\
\alpha=\frac{V_{o}}{V_{i}-V_{o}} \\
\text { Boost converter } \\
\alpha=\frac{V_{o}}{V_{i}}-1 \\
\text { Buck-boost converter } \\
\alpha=\frac{V_{o}}{V_{i}}\end{array}$ & $\alpha(1-x \bmod 1)$ \\
\hline Astable & $1-(a x+b) \bmod 1]$ \\
\hline Monostable & $\frac{1}{\beta} \ln \left[2-\mathrm{e}^{-\gamma(1-x \bmod 1)}\right]$ \\
\hline
\end{tabular}

In order to use equations (4) and (5) to calculate $A_{m}$, and hence $\left|A_{m}\right|^{2}$, the periodic spectral components, we now only need the invariant density $\rho$ of $F$, which exists provided that $\beta$ and $\gamma$ are such that $F$ is an expanding mapping. This condition is met when $|d F / d x|>1$ everywhere, which is equivalent to $\beta<\gamma /\left(2 \mathrm{e}^{\gamma}-1\right)$. We use $\beta=0.043$ and $\gamma=0.92$, corresponding to reference [7] with clock frequency $5 \mathrm{kHz}$; then $|d F / d x| \geq 5.4$ everywhere. When $x$ is just greater than an integer $F(x)$ attains its maximum value, $X$, which is $(1 / \beta) \ln \left[2-\mathrm{e}^{-\gamma}\right]$. The invariant density can now be calculated simply by iterating $F$ a large number of times and plotting a normalised histogram of the distribution of iterates on $[0, X]$. The result for $10^{6}$ iterations is plotted in figure 3. The final stage involves evaluating $\left\langle X_{0}\right\rangle$ from equation (5), using numerical integration, and then finding $A_{m}$ from equation (4). For the monostable multivibrator, the integrals with respect to $x$ can be done analytically, but, since $\rho(u)$ is not known explicitly, the integral with respect to $u$ is done numerically. The periodic spectral components, $\left|A_{m}\right|^{2}$, can then be found.

For the purposes of comparison the spectral calculation has been carried out in a number of different ways and the results are compared in figure 4. First of all, the periodic spectrum was computed by finding the sum in equation 1 for $N=5000$. The invariant density algorithm was also used. Finally, a segment of the monostable waveform was generated $(N=151$, corresponding to 1024 clock periods; 128 sample points per clock period) and a FFT algorithm used to estimate the PDS. Such a short segment was used because of the large number of sampling points required$2^{17}=131,072$.

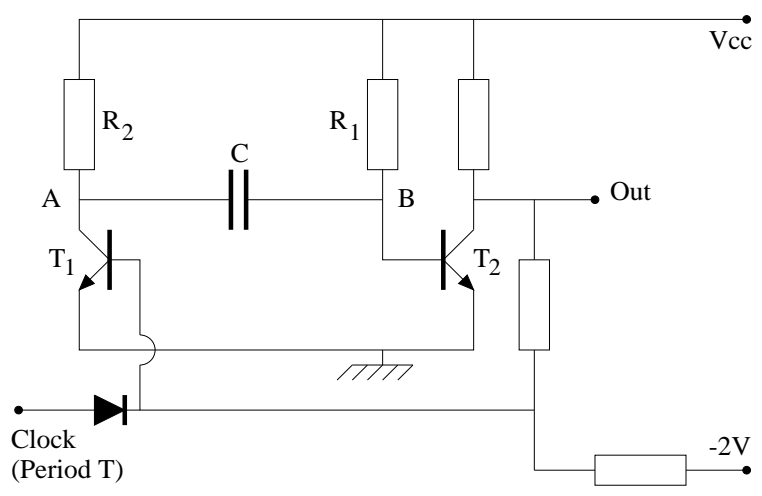

Figure 2: A monostable multivibrator.

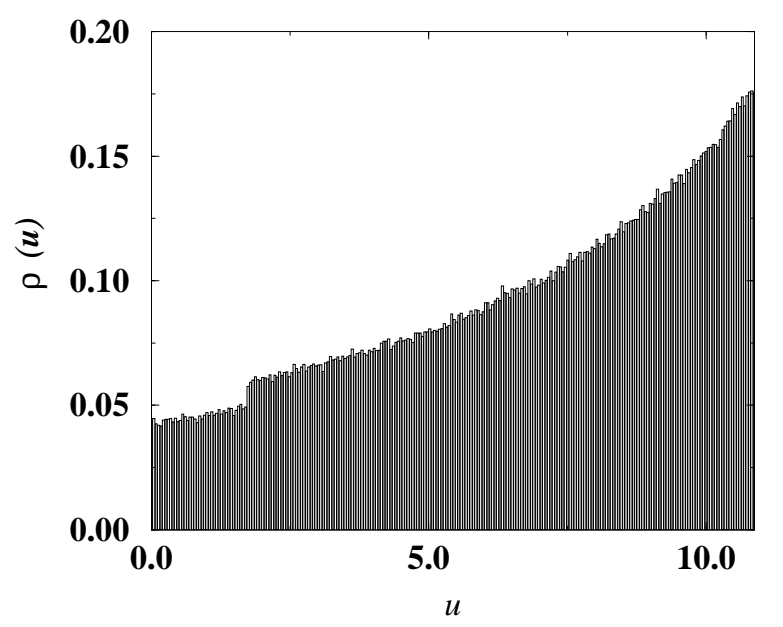

Figure 3: The invariant density of the monostable mapping $F(x)=(1 / \beta) \ln \left[2-\mathrm{e}^{-\gamma(1-x \bmod 1)}\right]$ with $\beta=0.043$ and $\gamma=0.92$.

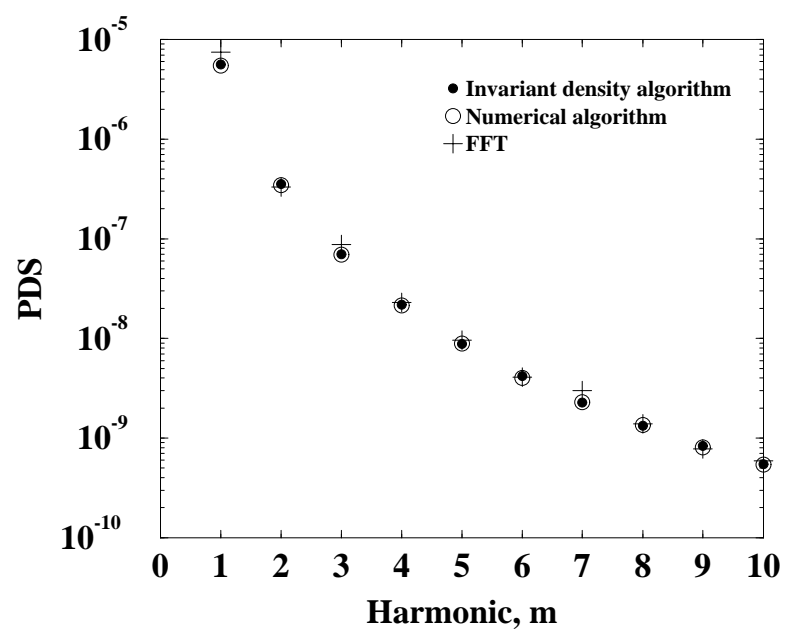

Figure 4: The PDS of the monostable waveform calculated in three different ways. 


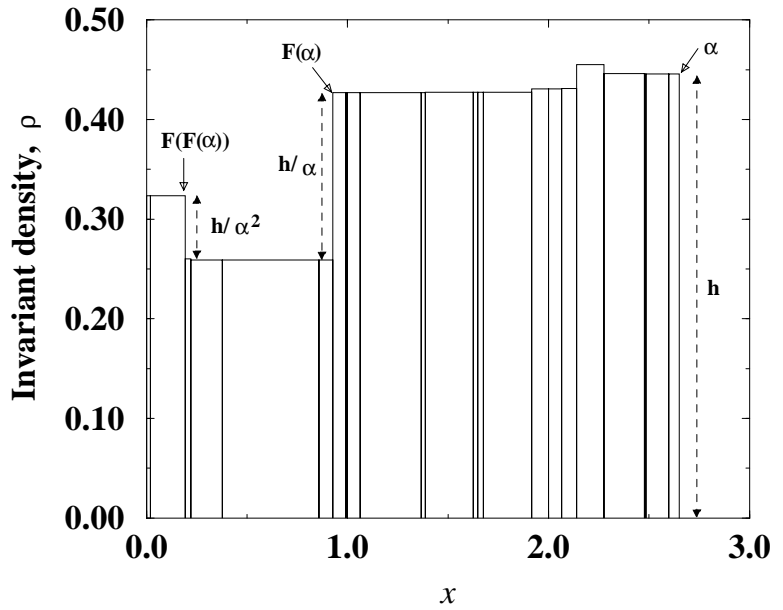

Figure 5: The invariant density of the boost converter mapping. It was calculated by the algorithm in [2] with $\alpha=$ 2.65 , and $h$ is chosen so that the density is normalised.

\section{DISCUSSION, FURTHER WORK AND CONCLUSIONS}

We have defined a class of clocked/autonomous two-state circuits whose behaviour can be described by a mapping $F$. Under certain conditions it is possible to calculate the periodic components of the PDS from the invariant density of $F$, when it exists. The degree to which this calculation can be carried out in closed form depends on the exact nature of $F$. For instance,

1. $F$ is Markov [8], i.e. $[0, X)$ can be partitioned into a finite number of intervals such that $F$ maps these intervals into each other. In this case, $\rho$ is a piecewise constant function over the intervals. The spectral calculation can be carried out entirely in closed form.

2. $F$ is piecewise linear but not Markov. This is true for the power converters mentioned earlier, for almost all values of $\alpha$. Here, $\rho$ is still piecewise constant, but there are an infinite number of partitions. The situation is illustrated in figure 5 for the boost converter mapping with $\alpha=2.65$, which shows how the mapping can be represented in 'almost closed form'.

3. $F$ is of some other form. The monostable mapping is an example of this. The invariant density has to be calculated numerically, as does equation 4 .

The class of systems that can be analysed in this way can clearly be extended, for instance to three or more states, and, for linear $y$ and $y^{\prime}$, ones in which the threshold $Y_{t}$ is time varying. The latter case would apply to converters with
PWM by natural sampling. Of interest too is the problem of calculating the PDS at frequencies other than the clock harmonics.

The possibility of switch-mode power supply interference reduction by chaos has already been mentioned [1], [2]. Of interest therefore is the behaviour of spectral peaks as circuit parameters are varied, and the algorithm for calculating these peaks presented here is ideal for spectral peak tracking.

\section{REFERENCES}

[1] J.H.B. Deane and D.C. Hamill, Improvement of power supply EMC by chaos, Electronics Letters, vol. 32 no. 12, page 1045 (June 1996)

[2] J.H.B. Deane, P. Ashwin, D.C. Hamill and D.J. Jefferies, Calculation of the periodic spectral components in a chaotic $d c$-dc converter, IEEE Transactions on Circuits and Systems - I: Fundamental Theory and Applications (1999)

[3] J.H.B. Deane, Chaos in a current-mode controlled boost $d c-d c$ converter, IEEE Transactions on Circuits and Systems, vol. 39 no. 8, pp 680-683 (1992)

[4] D.C. Champeney, "A handbook of Fourier theorems", Cambridge University Press, Cambridge, UK, ISBN 0521-265037 (1987)

[5] J-P. Eckmann and D. Ruelle, Ergodic theory of chaos and strange attractors, Reviews of Modern Physics, vol. 57 no. 3 part 1, pp 617-656 (1985)

[6] Y.S. Tang, A.I. Mees and L.O. Chua, Synchronisation and chaos, IEEE Transactions on Circuits and Systems, vol. 30, no. 9, pp. 620-626 (1983)

[7] J.H.B. Deane and D.J. Jefferies, Periodic, intermittent and chaotic states of clocked autonomous digital systems, International Journal of Circuit Theory and Applications, vol. 18, no. 2, pp. 175-187 (1990)

[8] S.H. Isabelle, "A signal processing framework for the analysis and application of chaotic systems", PhD Thesis, MIT (1995) 